\title{
Excited states of isolated fermions in the Higgs phase of gauge Higgs theo- ries
}

\author{
Jeff Greensite ${ }^{1, *}$ and Kazue Matsuyama ${ }^{1, * *}$ \\ ${ }^{1}$ Physics and Astronomy Dept., San Francisco State University, San Francisco CA 94132 USA
}

\begin{abstract}
We present evidence that seemingly elementary and non-composite objects, namely isolated static fermions in certain gauge Higgs theories, have a mass spectrum corresponding to localized excitations of the surrounding gauge and Higgs fields.
\end{abstract}

\section{Introduction}

Composite systems (molecules, atoms, nuclei, hadrons...) generally have a spectrum of excitations. What about noncomposite systems: charged "elementary" particles like quarks and leptons? If the particle is charged, then by Gauss's Law it is accompanied by a surrounding gauge (and possibly other) fields. If these surrounding fields interact with themselves, could they not also exhibit a spectrum of excitations? This would look like a mass spectrum of the isolated elementary particle. That doesn't happen in pure QED where the gauge field does not self-interact, and any energy eigenstate containing a static \pm charge pair is just the Coulomb field plus some number of photons. Gauge Higgs theories could be different.

\section{Pseudomatter operators}

One often reads that physical states are gauge invariant. This statement is almost, but not quite, true. The Gauss law constraint only requires invariance under infinitesimal gauge transformations. In QED, in an infinite volume, a physical state containing a single static charge transforms under a global subgroup of the gauge group. The ground state of pure QED containing a single static electric charge at point $\mathbf{x}$ is the Dirac state

$$
\begin{aligned}
\left|\Psi_{\mathbf{x}}\right\rangle & =\bar{\psi}^{+}(\mathbf{x}) \rho_{C}(\mathbf{x} ; A)\left|\Psi_{0}\right\rangle \\
\rho_{C}(\mathbf{x} ; A) & =\exp \left[-i \frac{e}{4 \pi} \int d^{3} z A_{i}(\mathbf{z}) \frac{\partial}{\partial z_{i}} \frac{1}{|\mathbf{x}-\mathbf{z}|}\right] .
\end{aligned}
$$

It is easy to check that $\left|\Psi_{\mathbf{x}}\right\rangle$ satisfies the Gauss Law. However, let $g(x)=e^{i \theta(x)}$ be an arbitrary $\mathrm{U}(1)$ gauge transformation, and we separate out the zero mode $\theta(x)=\theta_{0}+\tilde{\theta}(x)$. Then $\psi(\mathbf{x}) \rightarrow e^{i \theta(\mathbf{x})} \psi(\mathbf{x})$, but $\rho_{C}(\mathbf{x} ; A) \rightarrow e^{i \tilde{\theta}(x)} \rho_{C}(\mathbf{x} ; A)$. It follows that $\left|\Psi_{\mathbf{x}}\right\rangle \rightarrow e^{-i \theta_{0}}\left|\Psi_{\mathbf{x}}\right\rangle$ tranforms under a global subgroup of the local gauge symmetry group. Local symmetries cannot break spontaneously, of course (Elitzur).

\footnotetext{
*e-mail: greensit@sfsu.edu

**e-mail: kazuem@sfsu.edu
}

But global symmetries can. The operator $\rho_{C}(\mathbf{x} ; A)$ is a first example of what we refer to as a "pseudomatter" field [1]. It is responsible for dressing the bare charge with a Coulomb electric field.

In general, we define a pseudomatter field $\rho(\mathbf{x} ; A)$ to be a non-local functional of the gauge field which transforms like a matter field in the fundamental representation of the gauge group, except under the global center subgroup of the gauge group. In fact any $\mathrm{SU}(\mathrm{N})$ gauge transformation $g_{F}(\mathbf{x} ; A)$ to a physical gauge $F(A)=0$ can be decomposed into $N$ pseudomatter fields $\left\{\rho_{n}\right\}$, and vice-versa: $\rho_{n}^{a}(\mathbf{x} ; A)=g_{F}^{\dagger a n}(\mathbf{x} ; A)$. In particular, the operator $\rho_{C}^{*}(\mathbf{x} ; A)$ defined earlier is precisely the gauge transformation to Coulomb gauge in an abelian theory. Another example, of particular relevance to this work, is that any eigenstate $\xi_{n}(\mathbf{x} ; U)$ of the covariant Laplacian operator $-D^{2} \xi_{n}=\kappa_{n} \xi_{n}$ in a lattice $\mathrm{SU}(\mathrm{N})$ gauge theory is a pseudomatter field, where

$$
\begin{array}{r}
\left(-D^{2}\right)_{\mathbf{x y}}^{a b}=\sum_{k=1}^{3}\left[2 \delta^{a b} \delta_{\mathbf{x y}}-U_{k}^{a b}(\mathbf{x}) \delta_{\mathbf{y}, \mathbf{x}+\hat{k}}\right. \\
\left.-U_{k}^{\dagger a b}(\mathbf{x}-\hat{k}) \delta_{\mathbf{y}, \mathbf{x}-\hat{k}}\right]
\end{array}
$$

is the covariant Laplacian. This is the idea behind the Laplacian gauges of Vink and Wiese [2].

We may combine the Higgs field and pseudomatter fields with matter field creation operators to create physical states in gauge Higgs theories.

\section{Excited states of "elementary" particles}

For a static quark-antiquark pair in a pure gauge theory there is a tower of metastable states

$$
\Psi_{n}(R)=\bar{q}(\mathbf{x}) V_{n}(\mathbf{x}, \mathbf{y} ; U) q(\mathbf{y}) \Psi_{0}
$$

correpsonding to string excitations. This has been observed in computer simulations [3, 4]. For light quarks, the excited states lie on linear Regge trajectories, and these should also exist in the confinement region of a gauge 
Higgs theory. The question is whether, in the spin glass (aka Higgs) phase, there a similar tower of metastable states of the form

$$
\Psi_{n}(R)=\bar{q}^{a}(\mathbf{x})\left[\sum_{m} c_{m}^{(n)} \rho_{m}^{a}(\mathbf{x}) \rho_{m}^{\dagger b}(\mathbf{y})\right] q^{b}(\mathbf{y}) \Psi_{0},
$$

where the $\left\{\rho_{m}(\mathbf{x})\right\}$ are pseudomatter fields. We have investigated this question in several gauge Higgs models with results that we discuss briefly here. In the study of static fermion excitations, we find that each model has its own special features which must be taken into account.

There are various strategies that can be used to compute the spectrum of a static fermion-antifermion pair. For example, if $|\Psi(R)\rangle$ is some arbitrary physical state containing a static fermion-antifermion pair with separation $R$, and $E_{1}(R)$ is the lowest energy of such states above the vacuum energy $\mathcal{E}_{0}$, then on general grounds, as $T \rightarrow \infty$

$$
\left\langle\Psi\left|\mathcal{T}^{T}\right| \Psi\right\rangle=\sum_{n} c_{n} e^{-E_{n}(R) T} \rightarrow c_{1} e^{-E_{1}(R) T}
$$

where $\mathcal{T}$ is the transfer matrix multiplied by $e^{\varepsilon_{0}}$. The drawback of this method is that while it is easy to extract the ground state energy, it is not so simple to extract the energies of excited states. Alternatively, let $\left\{\left|\Phi_{\alpha}(R)\right\rangle\right\}$ span a subspace of the full Hilbert space with the two static charges. Then one could get an approximate spectrum by diagonalizing $\mathcal{T}$ in this subspace. This approach is followed in some lattice QCD spectrum calculations. Here the drawback is that this requires a pretty big set $\sim$ hundreds of states. This is not practical for our purposes, where it is computationally expensive to generate the $\left|\Phi_{\alpha}(R)\right\rangle$.

Our approach is as follows: Generate a small set of states $\left\{\left|\Phi_{\alpha}(R)\right\rangle\right\}$, and diagonalize either $\mathcal{T}$ or $\mathcal{T}^{p}$ in the small subspace spanned by these states. The hope is that one or more of the eigenstates $\left|\Psi_{n}\right\rangle$ in the subspace will be orthogonal (nearly) to the true ground state. If $|\Psi\rangle$ is such a state, then

$$
\left\langle\Psi\left|\mathcal{T}^{T}\right| \Psi\right\rangle=\sum_{n} c_{n} e^{-E_{n}(R) T} \rightarrow c_{e x} e^{-E_{e x}(R) T} \text { at large } T,
$$

where $E_{e x}$ is the energy of an excited state. Of course there are no guarantees this will work, it just has to be tried.

\section{Four Models}

\subsection{SU(3) gauge Higgs theory}

We begin with SU(3) gauge Higgs theory with a unimodular Higgs field in the fundamental color representation. Our calculations are carried out on a $14^{3} \times 32$ lattice volume, at $\beta=5.5$ with $\gamma=0.5$ and $\gamma=3.5$, in the confinement and Higgs phases respectively [5]. The action is

$$
\begin{aligned}
S= & -\frac{\beta}{3} \sum_{\text {plaq }} \operatorname{Re} \operatorname{Tr}\left[U_{\mu}(x) U_{v}(x+\hat{\mu}) U_{\mu}^{\dagger}(x+\hat{v}) U_{v}^{\dagger}(x)\right] \\
& -\gamma \sum_{x, \mu} \operatorname{Re}\left[\phi^{\dagger}(x) U_{\mu}(x) \phi(x+\widehat{\mu})\right]
\end{aligned}
$$

and the goal is to find excited states of static fermions in this system, if such states exist. Here (and in all gauge Higgs models discussed below) we impose the unimodular constraint $\phi^{\dagger} \phi=1$.

Let $\xi_{n}$ denote the eigenstates of the lattice Laplacian operator (no time derivatives) $-D^{2} \xi_{n}=\kappa_{n} \xi_{n}$, let $\mathcal{T}=e^{-\left(H-\mathcal{E}_{0}\right)}$ be the (rescaled) transfer matrix, and consider at each quark separation $R=|\mathbf{x}-\mathbf{y}|$ the 4-dimensional subspace spanned by three quark-pseudomatter states, and one quark-scalar state

$$
\begin{aligned}
\Phi_{n=1,2,3}(R) & =\left[\bar{q}^{a}(\mathbf{x}) \xi_{n}^{a}(\mathbf{x})\right] \times\left[\xi_{n}^{\dagger b}(\mathbf{y}) q^{b}(\mathbf{y})\right] \Psi_{0} \\
\Phi_{4}(R) & =\left[\bar{q}^{a}(\mathbf{x}) \phi^{a}(\mathbf{x})\right] \times\left[\phi^{\dagger b}(\mathbf{y}) q^{b}(\mathbf{y})\right] \Psi_{0} .
\end{aligned}
$$

We calculate numerically the matrix elements and overlaps

$$
\mathcal{T}_{m n}(R)=\left\langle\Phi_{m}|\mathcal{T}| \Phi_{n}\right\rangle \quad, \quad O_{m n}(R)=\left\langle\Phi_{m} \mid \Phi_{n}\right\rangle .
$$

The eigenvalues of $\mathcal{T}$ in the subspace are obtained by solving the generalized eigenvalue problem $[\mathcal{T}] \vec{v}_{n}=\lambda_{n}[O] \vec{v}^{(n)}$, and we have eigenstates of $\mathcal{T}$ in the subspace

$$
\left|\Psi_{n}(R)\right\rangle=\sum_{i=1}^{4} v_{i}^{(n)}\left|\Phi_{i}(R)\right\rangle
$$

Likewise we consider evolving states for Euclidean time $T$, and compute

$$
\begin{aligned}
\mathcal{T}_{n n}^{T}(R) & =\left\langle\Psi_{n}\left|\mathcal{T}^{T}\right| \Psi_{n}\right\rangle=v_{i}^{(n) *}\left\langle\Phi_{i}\left|\mathcal{T}^{T}\right| \Phi_{j}\right\rangle v_{j}^{(n)} \\
E_{n}(R, T) & =-\log \left[\frac{\mathcal{T}_{n n}^{T}(R)}{\mathcal{T}_{n n}^{T-1}(R)}\right]
\end{aligned}
$$

Integrating out the massive (i.e. static) fermion fields generates a pair of Wilson lines. The numerical computation of $\left\langle\Phi_{i}\left|\mathcal{T}^{T}\right| \Phi_{j}\right\rangle$ involves the expectation values of products of Wilson lines, terminated by matter or pseudomatter fields.

We have computed $E_{1,2}(R, T)$ in this model on a $14^{3} \times$ 32 lattice volume, at $\beta=5.5$ with $\gamma=0.5$ and $\gamma=3.5$, in the confinement and Higgs phases respectively. The results for $E_{n}(R, T)$ and the overlap for $\Psi_{1}(R), \Psi_{2}(R)$ in the Higgs phase, in the range $T=4-12$, are shown in Fig. 1. It is seen that the data has converged in this range of Euclidean time $T$.

There seems to be clear evidence of an excited state of a static fermion-antifermion pair, orthogonal to the ground state, whose energy is only weakly dependent on separation $R$. At large $R$ the fermions are understood to be isolated from one another. Because the energy gap is far smaller than the threshold for vector boson creation in this model, this excited state is stable.

\subsection{The $q=2$ abelian Higgs model}

This is a version of the abelian Higgs model in which the scalar field (like Cooper pairs) carries two units of electric charge:

$$
\begin{aligned}
S= & -\beta \sum_{\text {plaq }} \operatorname{Re}\left[U_{\mu}(x) U_{v}(x+\hat{\mu}) U_{\mu}^{*}(x+\hat{v}) U_{\nu}^{*}(x)\right] \\
& -\gamma \sum_{x, \mu} \operatorname{Re}\left[\phi^{*}(x) U_{\mu}^{2}(x) \phi(x+\widehat{\mu})\right] .
\end{aligned}
$$




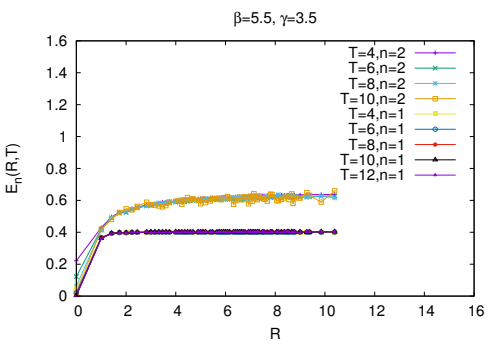

(a) Energies

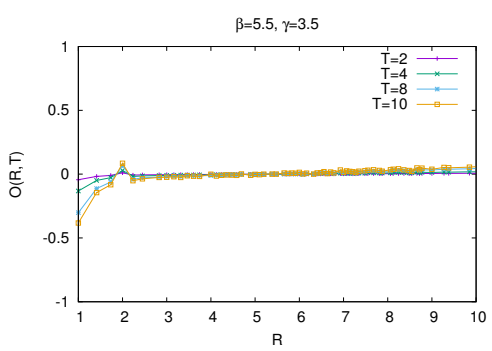

(b) Overlap

Figure 1. (a) Energies $E_{n}(R, T)$ of states $\Psi_{1}, \Psi_{2}$ after evolution for a period of $(T-1) / 2$ units of Euclidean time, at lattice couplings $\beta=5.5, \gamma=3.5$. Note the energy gap, which persists out to the largest $T$ values shown, of $E_{2}(R, T)-E_{1}(R, T) \approx 0.2$ in lattice units. (b) The overlap $O(R, T)$ between states $\Psi_{1}(R)$ and $\Psi_{2}(R)$ which are evolved (and then normalized) for $T / 2$ units of Euclidean time. $O(R, T)=0$ by construction at $T=0,1$, but the Euclidean time-evolved states are seen to remain approximately orthogonal for $R>2$ at larger $T$.

This theory has a phase diagram consisting of massless, Higgs, and confining phases, in this case separated by thermodynamic transition lines. The search for excited static fermion states was carried out in ref. [6] just inside the Higgs phase, at $\beta=3.0, \gamma=0.5$, and in this investigation the fermions were taken to have the same $q= \pm 2$ charges as the scalar field. An example of the relevant data is shown in Fig. 2(a). A surprise is that, following along the same lines of diagonalizing $\mathcal{T}$ in a small subspace, and judging by the fit to $\mathcal{T}_{11}(R, T)$ and $T_{22}(R, T)$, the lowest two states $\Psi_{1,2}$ seem to be very nearly exact eigenstates of the system, even at small $T$.

The ground and excited state energies, and also a rough estimate of the next excited state energy, vs. fermion separation are shown in Fig. 2(b). Once again, the first excitation is well below the threshold, and is therefore stable against massive photon emission. The second excited state, lying so near the threshold, most likely consists of the ground state plus a massive photon.

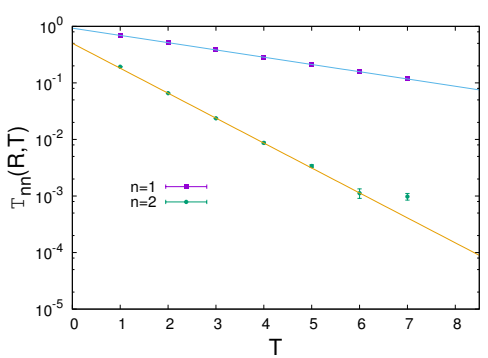

(a)

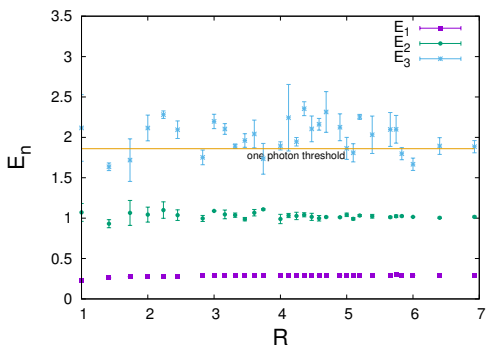

(b)

Figure 2. (a) An example of fits at $R=6.93$ on a $16^{4}$ lattice in the abelian Higgs model, with couplings $\beta=3, \gamma=0.5$. (b) Lowest, first excited, and second excited state energies vs. fermion separation.

\subsection{Effective Landau-Ginzburg model}

What about real superconductors? ${ }^{1}$ The effective LandauGinzburg model for ordinary superconductivity is a nonrelativistic $q=2$ abelian Higgs model of this form:

$$
\begin{aligned}
S= & -\beta \sum_{\text {plaq }} \operatorname{Re}\left[U U U^{*} U^{*}\right] \\
& -\gamma \sum_{x} \sum_{k=1}^{3} \phi^{*}(x) U_{k}^{2}(x) \phi(x+\hat{k}) \\
& -\frac{\gamma}{v^{2}} \sum_{x} \phi^{*}(x) U_{0}^{2}(x) \phi(x+\hat{t}) .
\end{aligned}
$$

where $v \sim 10^{-2}$ in natural units, is on the order of the Fermi velocity in a metal, and $\beta=1 / e^{2} \approx 10.9$ for ordinary electrodynamics. Go to unitary gauge, so that $U_{0}(x) \approx \pm 1$. We then compute the excitations around a pair of static $q= \pm 1$ (e) charges, having electrons, ions, and holes in mind. The parameters $\gamma, \beta$ determine the photon mass (inverse to the penetration depth) in lattice units, so for a given $\gamma$ the penetration depth fixes the lattice spacing in physical units. But this time things are not so simple, and diagonalizing $\mathcal{T}$ in a small subspace doesn't work. Eigenstates in the subspace flow in Euclidean time to the ground state.

What we do instead, at each separation $R$, is to diagonalize a power of the transfer matrix, $\mathcal{T}^{2 t_{0}}$, in the basis $\Phi_{\alpha}$,

\footnotetext{
${ }^{1}$ This is work in progress, so we display only preliminary results.
} 
so that

$$
\left\langle\Psi_{m}\left|\mathcal{T}^{2 t_{0}}\right| \Psi_{n}\right\rangle=\lambda_{n}\left(t_{0}\right) \delta_{m n},
$$

and define $\Psi_{n}(t)=\mathcal{T}^{t} \Psi_{n}$. Suppose, after evolving $\Psi_{1}$ by $t_{0}$ units of Euclidean time, that $\Psi_{1}\left(t_{0}\right)$ is approximately the true ground state in the full Hilbert space. It follows that all $\Psi_{n>1}\left(t_{0}\right)$ are orthogonal to the ground state, simply because $\left\langle\Psi_{m}\left(t_{0}\right) \mid \Psi_{n}\left(t_{0}\right)\right\rangle \propto \delta_{m n}$, and therefore, at large $T>2 t_{0}$

$$
\begin{aligned}
\mathcal{T}_{22}(R, T) & =\left\langle\Psi_{2}\left|\mathcal{T}^{T}\right| \Psi_{2}\right\rangle \\
& =\left\langle\Psi_{2}\left(t_{0}\right)\left|\mathcal{T}^{T-2 t_{0}}\right| \Psi_{2}\left(t_{0}\right)\right\rangle \\
& \rightarrow \text { const } \times e^{-E_{e x} T} \text { where } E_{e x}>E_{1}
\end{aligned}
$$

For this calculation we choose $2 t_{0}=9$ and $\gamma=0.25$. Sample results at separation $R=5.385$ are shown in Fig. 4 . We

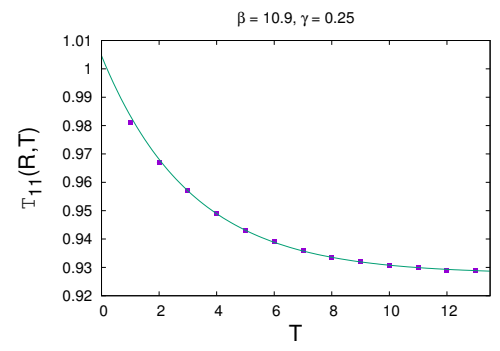

(a)

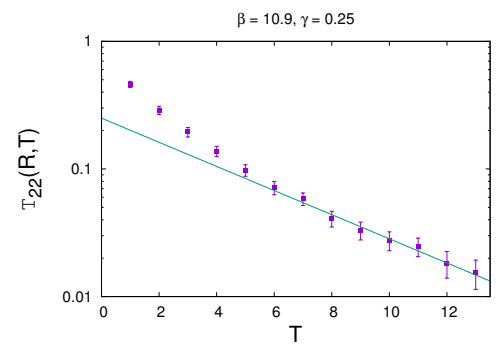

(b)

Figure 3. $\mathcal{T}_{n n}(R, T)$ at $R=5.385$ vs $T$ in the effective LandauGinzburg model of superconductivity.

fit $\mathcal{T}_{11}$ to

$$
f_{1}(T)=a_{1} \exp \left(-b_{1} T\right)+c_{1} .
$$

The fact that $c_{1} \neq 0$ means that the ground state energy $E_{1} \approx 0$, while $b_{1}$ gives an excited state energy. Then we fit $T_{22}$ in the range $T>6$ to a single exponential $f_{2}(T)=a_{2} \exp \left(-b_{2}\right)$, and the coefficient $b_{2}<b_{1}$ gives another excitation energy. The data at $R<4.0$ are rather noisy, with large $\chi^{2}$. The results for $R>4$ are shown in Fig. 4. Once again, the first excited state is stable. The next excited state, which is right on the threshold, is presumably the ground state plus a massive photon. The question of whether such new states could be detected experimentally, e.g. by ARPES, is of course of great interest.

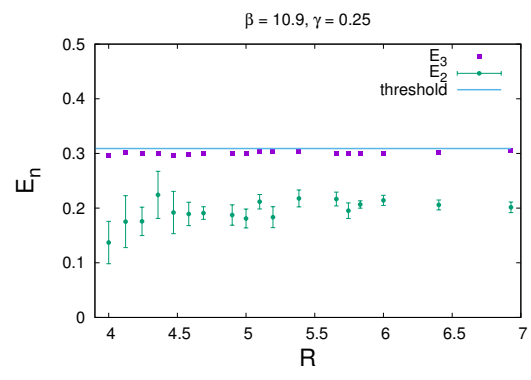

Figure 4. Ground state and excitation energies in the LandauGinzburg model. The ground state energy is $E_{1} \approx 0$.

\section{Excitations in chiral gauge theories}

The electroweak sector of the standard model is a chiral gauge theory, and unfortunately there is no known lattice formulation of chiral non-abelian gauge theories with a continuum limit. There is a formulation for U(1) gauge theories due to Lüscher, involving overlap fermions. It is, however, difficult to implement numerically. So in this exploratory work, we chose a simpler option: only static fermions in a quenched formulation of a U(1) lattice gauge theory, with oppositely charged right and lefthanded fermions, using a version of the old Smit-Swift lattice action [7, 8]. Lattice doublers restore chiral symmetry, so the original idea was to use a Wilson-style non-local mass term so that the mass of the doublers is infinite in the continuum limit. It is known that the continuum limit doesn't work [9], and the Smit-Swift formulation is not a true chiral gauge theory. Moreover, the positivity of the transfer matrix is unproven. But at least, at finite lattice spacing, there is a mass asymmetry between the desired states and the doublers in part of the phase diagram. So this is a model that can be studied with our methods.

The calculation is a little more involved than in the previous three models, and instead of a Wilson line there is a more complicated covariant object which connects to the pseudomatter fields. We leave the details of this computation to ref. [10], and here only display the results. The numerical simulation is carried out on a $14^{3} \times 32$ lattice volume at $\beta=3.0, \gamma=1.0$ using $n_{e v}=4$ Laplacian eigenstates, along with combinations of the $q=2$ Higgs field and pseudomatter fields. Results are shown in Fig. 5, where $\lambda_{n}(R, T) \equiv \mathcal{T}_{n n}(R, T)$. In these figures, $E_{1}$ is derived from $\lambda_{1}, E_{2}$ is derived from either $\lambda_{3}$ or $\lambda_{4}$ in the large $T \geq 4$ range, and $E_{3}$ is derived from $\lambda_{2}$ in the small $T \leq 5$ range. Energies $E_{1,2,3}$ vs. $R$ are displayed in Fig. 6.

\section{Conclusions}

We have found that in all four gauge Higgs models that we have investigated, the gauge+Higgs fields surrounded a charged static fermion have a spectrum of localized excitations, which cannot be interpreted as simply the ground state plus some massive bosons. This means that charged 


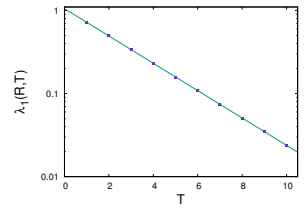

(a)

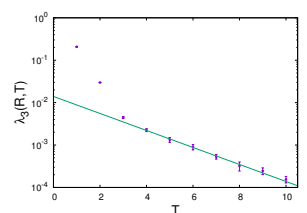

(c)

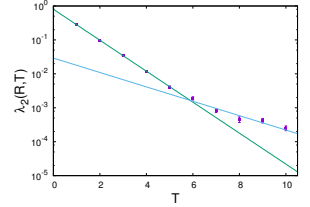

(b)

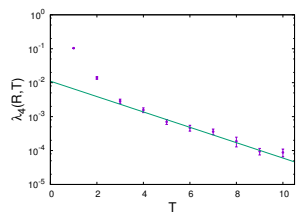

(d)
Figure 5. $\lambda_{n}(R, T)=\mathcal{T}_{n n}(R, T)$ vs. $T$ at $R=4.243$ in a chiral gauge theory at finite lattice spacing with Smit-Swift regularization.

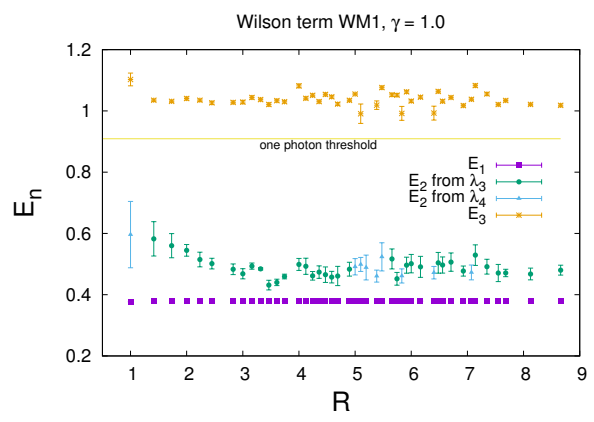

Figure 6. Ground and excitation energies of static fermions in the chiral gauge model at $\beta=3, \gamma=1$, shown together with the threshold for creation of a massive photon above the ground state energy. "elementary" particles, which are not usually thought of as composite objects, can have a mass spectrum in gauge Higgs theories. This conclusion seems robust. We see it in SU(3) gauge Higgs theory, the abelian Higgs and LandauGinzburg models, and also in chiral U(1) models. The question now is whether such excitations can be observed experimentally in the gauge Higgs models that we know about, i.e. superconductivity and the electroweak sector of the standard model. We reserve this interesting question for future investigation.

\section{References}

[1] J. Greensite, K. Matsuyama, Phys. Rev. D96, 094510 (2017), 1708.08979

[2] J.C. Vink, U.J. Wiese, Phys. Lett. B289, 122 (1992), hep-lat/9206006

[3] K.J. Juge, J. Kuti, C. Morningstar, Phys. Rev. Lett. 90, 161601 (2003), hep-lat/0207004

[4] B.B. Brandt, M. Meineri, Int. J. Mod. Phys. A 31, 1643001 (2016), 1603.06969

[5] J. Greensite, Phys. Rev. D 102, 054504 (2020), 2007.11616

[6] K. Matsuyama, Phys. Rev. D 103, 074508 (2021), 2012. 13991

[7] J. Smit, Acta Phys. Polon. B 17, 531 (1986)

[8] P.V.D. Swift, Phys. Lett. B 145, 256 (1984)

[9] M.F.L. Golterman, D.N. Petcher, J. Smit, Nucl. Phys. B 370, 51 (1992)

[10] J. Greensite, Phys. Rev. D 104, 034508 (2021) 